\title{
A METHOD FOR DETECTING BONE DENSITY AT DIFFERENT SIDES AND FIVE SITES OF MANDIBULAR BONE BY NEW COMPUTERIZED MEASUREMENTS
}

\author{
Mona Moustafa Ebrahim Elramady
}

\begin{abstract}
Introduction: The bone quality also affects the stress distribution under buccolingual load. Low density bone presents reduced stiffness and increases implant displacement. Under highest displacement, the bone is deformed, consequently higher stresses in the bone are predicted. The mental foramen is a principle factor for placing implants in the foraminal rang of the mandibular arch.
\end{abstract}

Objectives: To detect and identification of mandibular bone density around the tooth and alveolar ridges at the different five sites for both male and female groups and comparing them for accurate plane and not interfere with the mental foramen and canal during surgical treatment.

Material and Methods: Twelve patients ranged from 45-60 year old (6 males and 6 female) came to the Department of Prosthodontics, Alexandria University. Studied for identify the accurate nature of bone structures and detecting measurement of bone density around the implant prior to their surgical treatment. At the insertion after delivery of the denture and comparing between two gender and age groups) the evaluation of alveolar mandibular bone density using a new computerized method (Image J program) which was not used in the previous studies for prober assessment of bone height measurements.

Results: The result of bone density measurements around 5 sites of mandibular bone, the mean and standard deviation of bone density measurements in right side for both male and female groups and statistical results comparison were done. There was insignificant change of bone density around the 5 sites at both groups. In the midline, there was gradual increase in the bone density from midline region to molar region and in males more than females.

Conclusions: There was no statistical significant difference between the right side for female and male density at different sites, indicating more favorable stable supported overdenture with the use of accurate surgical position.

KEYWORD: Panoramic x-ray, bone density, new computerized measurement, Mandibular bone, overdenture

\footnotetext{
* Lecturer at Prosthodontics Department, Pharos University, Alexandria City, Alexandria, Egypt.
} 


\section{INTRODUCTION}

The stress distribution under buccolingual load is influenced by bone quality. Low density bone presents leads to decrease stiffness and also increases implant displacement. When the displacement is greater, the bone is deformed and resulting in the expectation of higher stresses in the cortical and cancellous bone. The mental foramen is a critical anatomical landmark when inserting implant in the foraminal area of the mandibular arch and the possibility of presence of differences in the number of foramina and its sites and the location of anterior loop of mental nerve to be found mesial to mental foramen should be considered before treatment. In this study, we use new methods to provide the measurements of bone density record around the tooth and alveolar bone at 5 sites and recording the average and mean around this 5 sites for both groups (males and females).

Before incorporating a dental prosthetic treatment into treatment plan, several factors must be taken into consideration. In a previous study, the use of periapical and panoramic radiographic films were introduced for implant and several treatment planning. Recently, 3-dimensional imaging has become famous used and is often used in dentistry. The cone beam computerized tomography (CBCT) scan gave 3-dimensional images of maxillary and mandibular morphology, and so giving multiple views with minimal radiation can be produced, which leading to interactive treatment planning. By the providing a stronger visualization of the anatomy and morphology of the surgical site, before surgery as a diagnostic imaging aid permitting the dentist to provide a principle factor for measuring the average of remaining bone found at any present area and provide a stronger information about any anatomical structures in the mouth ${ }^{(1)}$.

Pre-operative surgical planning is a principle factor for successful dental implant (DI) therapy. The selection of a suitable imaging modality together with the expert's judgment provides the suitable clinical scenario aids in the treatment planning. The functional aesthetics present at the location of missing dentition referred to be observed in the patient's aesthetic need, anatomical differences and patient's systemic condition. Various radiographic methods give insight important anatomical structures especially in the mandibular arch which play an achieve factor in any surgical treatment concerning to osteotomy, nerve repositioning and ridge split for implant placement ${ }^{(2)}$. It is a very important need for the dentist to study bone density, thickness and integrity of the cortical bone condition and also the optimal position and also the exact needed position and determination of the DI in addition to the important vital anatomic structures.

Three-dimensional (3D) digital radiography has been used perfectly and with increase success rates in clinical practice ${ }^{(3,4)}$. The value of cone beam computed tomography (CBCT) imaging that have a major factor for analyzing morphologic structure related to the head and neck which were concerned to the studying of alveolar bones and nerve canals ${ }^{(2)}$. Selecting the osteotomy site and the thickness of alveolar bone to be used in the treatment is a principle factor because of its near position to the inferior alveolar nerve (IAN), mental foramen (MF) and maxillary sinuses ${ }^{(5,6)}$.

\section{MATERIALS AND METHOD}

To evaluate the average of the different bone density at different tooth locations. Twelve patients ranged from 45-60 year old (6 males and 6 female) came to the Department of Prosthodontics, Alexandria University with past medical history. The measurement were done at various sites related to molar (FM), premolar (FP), and anterior near canine (FC) regions and mental foramen line and midline as a reference points for edentulous and dentate sites also were registered. At insertion of the denture and comparing between two gender and age groups) the evaluation of alveolar bone density using a new computerized method (Image $\mathbf{J}$ program) which was not used in the previous studies for prober assessment of bone height measurements. 


\section{Methods}

Using a new computerized method (Image $\mathbf{J}$ program) data set from the CBCT was uploaded to the dynamic navigation system (X-Guide, X-Nav Technologies) and introduced into planning system related to it. The software allows for nerve surveying and implant-dimension utilized with several views to position the inserted implant into the selected site of bone. Files from computerized Image J program can be measured and compared by taken the mean and standard deviation statistical analysis.

To detect the marginal bone density around each reference sites before surgery: On the images taken by the computerized Image $\mathrm{J}$ program around each sites for every case and comparing the results of male and female. A line was drawn from the crest of the alveolar ridge and extends to surround each site in rectangular shape around each socket and above the mental foramen site by $2 \mathrm{~mm}$ and all the results was tabulated, collected and compared between the two groups. (Figure 1)

\section{RESULTS}

Comparison of subject characteristics as (age, sex and density) were recorded and compared for all the subjects and the mandibular measurements were compared between them. (Table 1)

The means and standard deviation for the bone density for females before treatment at midline (ML), canine (FC), first premolar (FP), Mental foramen (MF) and first molar (FM) for the right side was $155.9 \pm 33.9,157.3 \pm 27.6,139.5 \pm 33,129.6 \pm 43.3$ and $128 \pm 26.14$ while for the males it was resulted that $134.7 \pm 40.8,155 \pm 27,134.1 \pm 21.3,126.1 \pm 29.7$ and 135.5 \pm 26.21 . (Table 2)

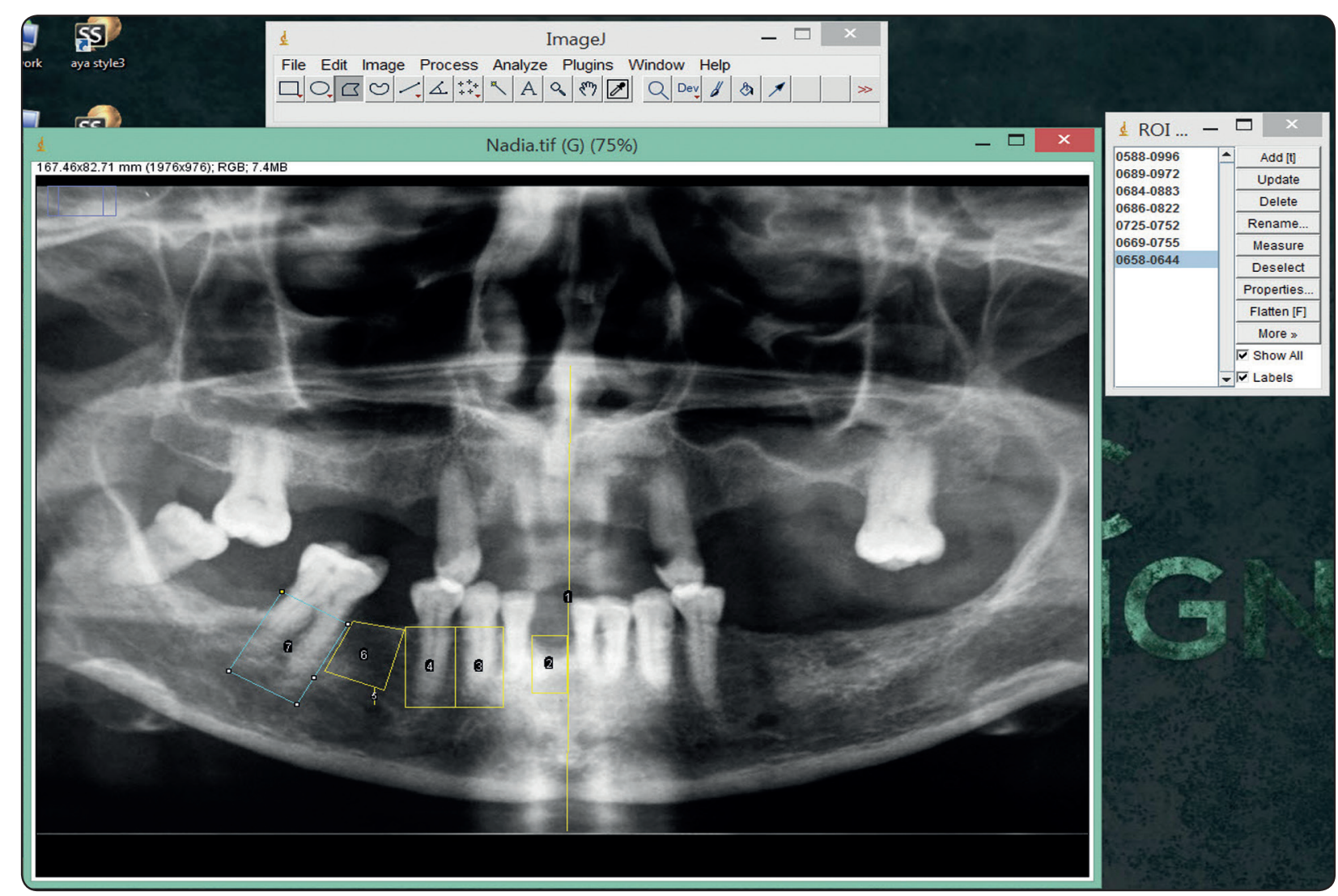

Fig. (1) Bone density assessment at different sites of mandibular bone 
The least bone density at all the evaluated sites of the alveolar bone level was found in the midline site of the mandibular bone and there was gradual increase in the bone density from midline site to molar site.

According to the differences in the age and gender of the studied patients, it was found that higher mean bone densities value of the evaluated sites was found to be increased in males than that for females and this may be according to the hormonal effect in female and also the presence of higher bone mass in male.
TABLE (1): Distribution of the studied cases according to gender and age $(n=12)$

\begin{tabular}{|c|c|}
\hline & No. $(\%)$ \\
\hline Gender & \\
\hline Male & $6(50 \%)$ \\
\hline Female & $6(50 \%)$ \\
\hline Age (years) & \\
\hline Mean \pm SD. & $49.75 .7 \pm$ \\
\hline Median (Min. - Max.) & $47(45-59)$ \\
\hline
\end{tabular}

TABLE (2): Comparison between females and males according to bone density five positions (right side)

\begin{tabular}{|l|c|c|c|c|}
\hline \multicolumn{1}{|c|}{$\begin{array}{c}\text { Bone density five } \\
\text { positions (right side) }\end{array}$} & $\begin{array}{c}\text { Female } \\
(\mathbf{n = 6}\end{array}$ & $\begin{array}{c}\text { Male } \\
(\mathbf{n = 6})\end{array}$ & $\mathbf{t}$ & $\mathbf{p}$ \\
\hline At midline & $155.9 \pm 33.9$ & $134.7 \pm 40.8$ & 0.980 & 0.350 \\
\hline At canine & $157.3 \pm 27.6$ & $155 \pm 27$ & 0.150 & 0.884 \\
\hline At first premolar & $139.5 \pm 33$ & $134.1 \pm 21.3$ & 0.337 & 0.743 \\
\hline At mental formena & $129.6 \pm 43.3$ & $126.1 \pm 29.7$ & 0.164 & 0.873 \\
\hline At first molar & $128 \pm 26.14$ & $135.5 \pm 26.21$ & 0.491 & 0.634 \\
\hline
\end{tabular}

Data was expressed by mean \pm SD.

p: $p$ value for comparing between males and females

\section{DISCUSSION}

Sghaireen et al. ${ }^{(6)}$, concluded that the mandibular premolar lingual area as regardless of the dentition status, a minimum of $6 \mathrm{~mm}$ bone is needed for placing the dental implant, the evaluation of surgical site with $\mathrm{CBCT}$ should be evaluated before surgery and became a principle factor before implant placement. It was found that the IAN is inserted more lingually in the molar site and directed to the buccal side as it directed to the premolar region. There were small differences found in the gender, age and side in the difference dental cases as regards to the dimensions of buccal, lingual and coronal bone available around the IAN.

\section{t: Student t-test}

The using dynamic navigation in a previous study for the evaluation of position and accuracy for implant placement. They concluded that determined navigation can provide exact implant placement at the same accuracy of static guide and is more advanced over free head implant positioning and also there was a learning curve to achieve it perfectly ${ }^{(7)}$.

Mathew et al. ${ }^{(8)}$, found that insertion of the implants at the molar area of the mandible may leads to opening of the lingual cortex penetration as a results of excessive inclined ridge or excessive lingual concavity. Existence of mental loop and anterior mandibular canal in the mandibular area needs perfect assessment using several tomographic slices. 
Spielau et al ${ }^{(9)}$, studied the using of a second surgical guide to be adjusted on previously secured position of implants can transfer the implant in the extraction sites after the positioning a first toothsupported surgical guide with the extracted of the related supporting teeth with a guided approach.

Mistry et al. ${ }^{(10)}$, concluded that patients with low glycemic control will have excessive residual ridge resorption and also bone resorption at the implant insertion sites is statistically similar in diabetics when compared to nondiabetics.

Yoon et al. ${ }^{(11)}$, concluded that preoperative imaging is imperative to the success of implant prosthetic positioning together with a good treatment planning and evaluation of the surgical site, a CBCT scan is a perfect diagnostic tool that can gave important information about the related anatomical and morphological differences in the sites of concern.

The presence of vital anatomical mandibular structures such as the inferior alveolar nerve canal and the inferior border of the anterior mandible were help as significant points in data gathering. The lingual concavity should be calculated and carefully determined to be informed if the implant positioning is recommended in the posterior first molar area of the mandible ${ }^{(11)}$.

The anterior region of the mandible contain the sublingual and submental arteries which is determined by the lingual concavity must be avoided to decrease the risk of accidental hemorrhage and the airway obstruction as a result of its near to the mandible which is determined by using CBCT. The preoperative diagnostic measures must be evaluated to ensure exact implant placement to avoid potential life-threatening problems ${ }^{(11)}$.

Kenawy et al., in $2017^{(12)}$, compared bone resorption and bone density between diabetic and nondiabetic patients and found that no significant difference recorded between bone quantity, there was decreased bone density in diabetic patients. Taylor et al., in $1998^{(13)}$, supporting the hypothesis that noninsulin-dependent diabetes mellitus patients have increase progression of alveolar ridge resportion and other studies supports these findings as the ORI whose found to be decrease in diabetic patients as compared to nondiabetics.

Tadiparthi and Sujatha in $2016^{(14)}$ concluded that glycemic control is an effective predetermined factor of bone resorption and so the diabetic patients were found to record higher increase in the alveolar bone resorption related to the right and left premolar region $^{(15)}$.

Methods for assessed bone density clinically such as standard radiography, dualenergy X-ray absorptiometry, digital image analysis, ultrasound, and CT while CT is considered an effective noninvasive system for gain bone images before to dental implant positioning. Quantitative computed tomography (QCT) has the greater advantage of qualifying. It allows the localization of precise three-dimensional anatomic and direct density measurements, expressed in $\mathrm{HU}^{(16)}$.

The separation found at the midline symphysis menti is slowly removed between the 4 th and $12^{\text {th }}$ months after birth. As a result of the growing of the mandible in two halves of mandibular bodies. The reasons of decrease density in this site are due to the synostosis joint present in this site ${ }^{(17)}$.

As a results of spread of occlusal force with the mastication mechanism. The maximum forces are found to be more from the anterior towards the posterior teeth ${ }^{(18)}$. And so it was detected in both jaws that the bone density increased gradually from the midline toward the posterior region.

It was suggested that the mandibular and so the posterior area found for the mandibular jaw contains denser and thicker cortical bone.

The presence of thick oblique ridges together with the attachment of mastication muscle leading to the greatest density at the retromolar area in the mandibular jaw. And also, include denser and thicker cortical bone which show slowly increase from the anterior to the posterior area ${ }^{(19,20)}$. 
It was explained that the difference in loads expressed as (compression, tension, and torsion) between the maxilla and mandible were leads to the mandible have much increase in the bone density measurements than the maxilla, which could be ${ }^{(21)}$ And this result agrees with Park et al ${ }^{(22)}$.

In a Korean population with the exception that they observed the highest bone density measurements were in the canine premolar region of the maxilla.

In general, it was found that the bone density values recorded were found to be more elevated in an Indian population than in a Korean population ${ }^{(22,23)}$. Especially in the canine premolar resion of the maxilla. Lifestyle factors which are found to influence bone density such as dietary calcium intake, physical activity, smoking, and alcohol intake ${ }^{(24)}$. Other possible cause was the variation in the observed bone mineral density evaluation is the difference in methodological factor such as the use of different slice thickness, software, CT machines, etc. ${ }^{(25)}$

So, it is important to detect the exact site of bone density before planning the incursion of miniimplants at the site of interest.

It was found that the average of tooth movement is inversely related to the bone density. When the density was lowered, the average of tooth movement will be increases ${ }^{(21)}$. And the bone supporting the mandibular molars was having more denser bone that that supported the maxillary.

The average of tooth movement increase while the a of bone density decrease and the later mesial drift when occur the roots engaged the high density bone by moving roots together with the average of tooth movement decrease. So, the low density region need the use of anchorage by transpalatal, implant, etc. is fundamental ${ }^{(21)}$.

Bone density is considered a principle factor for determination of the exact site of mini-implant that takes place by mechanical means of retention more than osteointegration. The smaller area of bone contacting to the implant body, the more elevated of the overall stress, when all the giving factors are equal ${ }^{(23,26)}$.

So, the success of the dental implant treatment is affected by the elevated of drilling temperature which leading to the occurrence of bone necrosis and this must be avoided during insertion of many implant into posterior part of mandibular bone to avoid the generated elevated temperature the dentist must avoid control the generation of elevated drilling temperature by the use of profuse irrigating saline solution. Also, avoid both the applied pressure on bone together with avoid using on old warn drill ${ }^{(27)}$.

\section{CONCLUSIONS}

- The least bone density of the buccal and lingual cortical plates at the alveolar bone level was found in the midline area of the mandibular bone and there was gradual increase in the value of the bone density from midline region to molar area.

- At the level of basal cortical bone, the area between first and second molar area found to record the highest bone density measurements and then followed by the area between the second premolar and first molar and then decrease gradually from the area of premolars and the canine and anterior teeth till reaching the midline area.

- The knowledge of the nature of bone density in the mandibular helps to reach to the following evaluation:

Provide the exact information between different clinical collected data.

Allow the prosthodontics to determine or proper strategies for the exact position of the implant required placement with the required precautions

- Completion of other future studies to assess the bone density at many sites of the basal to alveolar bones. 
- Also, future studies to use CT and the standard radiographic methods for the evaluation of the comparing of bone densities at the insertion sites of the mini-implant.

\section{REFERENCES}

1. Horner K, O’Malley L, Taylor K, Glenny AM. Guidelines for clinical use of CBCT: a review. Dentomaxillofac Radiol. 2015;44:20140225.

2. Weiss R, Read-Fuller AM. Cone beam computed tomography in oral and maxillofacial surgery: An evidence-based review. Dent J. 2019;7:52.

3. Benavides E, Rios HF, Ganz SD, An CH, Resnik R, Reardon GT, et al. Use of cone beam computed tomography in implant dentistry: the International Congress of Oral Implantologists consensus report. Implant Dent. 2012;21:78-86.

4. Srivastava KC, Ijar Shrivastava D, Austin D. Journey towards the $3 \mathrm{~d}$ dental imaging- the milestones in the advancement of dental imaging. Int J Adv Res. 2016;4:377-82.

5. Jacobs R, Salmon B, Codari M, Hassan B, Bornstein MM. Cone beam computed tomography in implant dentistry: Recommendations for clinical use. BMC Oral Heal. 2018; $18: 88$.

6. Sghaireen MG, Srivastava KC, Shrivastava D, Ganji KK, Patil SR, Abuonq A, et al. A CBCT based three-dimensional assessment of mandibular posterior region for evaluating the possibility of bypassing the inferior alveolar nerve while placing dental implants. Diagnostics. 2020;10:406.

7. Block MS, Emery RW, Lank K, Ryan J. Implant placement accuracy using dynamic navigation. Int J Oral Maxillofac Implants. 2017;32:92-9.

8. Mathew A, Sadiq RM, Ergieg S, al Shayeb M, Abuhijleh E, Varma SR, et al. Pre-treatment implant CBCT analysis of anterior mental loop length, lingual concavity and inclination of alveolar ridge in the mandible-A UAE study. Indian J. Forensic Med. Toxicol. 2020;14:4181.

9. Spielau T, Hauschild U, Katsoulis J. Computer-assisted, template-guided immediate implant placement and loading in the mandible: a case report. BMC Oral Health. 2019;19:55.

10. Mistry RA, Pisulkar SK, Godbole S, Sathe S, Borle A. An appraisal of bone resorption in completely edentulous diabetic and nondiabetic patients at prospective implant site in anterior mandible using digital volumetric tomography and its correlation with glycemic control: A case-control study. Natl J Maxillofac Surg. 2020;11:28-33.

11. Yoon TY, Patel M, Michaud RA, Manibo AM. Cone beam computerized tomography analysis of the posterior and anterior mandibular lingual concavity for dental implant patients. J Oral Implantol. 2017;43:12-8.

12. Kenawy S, Hegazy R, Hassan A, El-Shenawy S, Gomaa $\mathrm{N}$, Zaki $\mathrm{H}$, et al. Involvement of insulin resistance in D-galactose-induced age-related dementia in rats: Protective role of metformin and saxagliptin. PLoS One. 2017;12:e0183565.

13. Taylor GW, Burt BA, Becker MP, Genco RJ, Shlossman M, Knowler WC, et al. Non-insulin dependent diabetes mellitus and alveolar bone loss progression over 2 years. J Periodontol. 1998;69:76-83.

14. Tadiparthi DJ, Sujatha D. Evaluaton of vertical bone heights of maxillary and mandibular residual ridges among edentulous diabetics by digital orthopantomograph. Int $\mathbf{J}$ Curr Res. 2016;8:5.

15. Mistry RA, Pisulkar SK, Godbole S, Sathe S, Borle A. An appraisal of bone resorption in completely edentulous diabetic and nondiabetic patients at prospective implant site in anterior mandible using digital volumetric tomography and its correlation with glycemic control: A case-control study. Natl J Maxillofac Surg. 2020;11:28-33.

16. Lindh C, Nilsson M, Klinge B, Peterson A. Quantitative computed tomography of trabecular bone in the mandible. Dentomaxillofac Radiol. 1996; 25:146-50.

17. Sperber GH. Craniofacial Development. London: BC Decker; 2001.

18. Johnsen SE, Svensson KG, Trulsson M. Forces applied by anterior and posterior teeth and roles of periodontal afferents during hold-and-split tasks in human subjects. Exp Brain Res. 2007;178:126-34.

19. Kim JH, Joo JY, Park YW, Cha BK, Kim SM. Study of maxillary cortical bone thickness for skeletal anchorage system. J Korean Oral Maxillofac Surg. 2002;28:249-55.

20. Lim JE, Lim WH, Chun YS. Cortical bone thickness and root proximity at mandibular interradicular sites: implications for orthodontic mini-implant placement. Korean J Orthod. 2008;38:397-406.

21. Roberts WE. Bone physiology, metabolism, and biomechanics in orthodontic practice. In: Graber TM, Vanarsdall RL, Vig KWL, (eds). Orthodontics: Current Principles and Techniques. $4^{\text {th }}$ ed. St Louis: Mosby; 2005. pp 221-92. 
22. Park HS, Lee YJ, Jeong SH, Kwon TG. Density of the alveolar and basal bones of the maxilla and the mandible. Am J Orthod Dentofacial Orthop. 2008;133:30-7.

23. Chun YS, Lim WH. Bone density at interradicular sites: implications for orthodontic mini-implant placement. Orthod Craniofac Res. 2009;12:25-32.

24. Ettinger B, Sidney S, Cummings SR, Libanati C, Bikle DD, Tekawa IS, et al. Racial differences in bone density between young adult black and white subjects persist after adjustment for anthropometric, lifestyle, and biochemical differences. J Clin Endocrinol Metab. 1997;82:429-34.
25. de Oliveira RCG, Leles CR, Normanha LM, Lindh C, Rotta-Ribeiro RF. Assessments of trabecular bone density at implant sites on CT images. Oral Surg Oral Med Oral Pathol Oral Radiol Endod. 2008;105:231-8.

26. Hedia HS. Stress and strain distribution behavior in the bone due to the effect of cancellous bone, dental implant material and the bone height. Biomed Mater Eng. 2002;12:111-9.

27. Tehemar SH. Factors affecting heat generation during implant site preparation: a review of biologic observations and future considerations. Int J Oral Maxillofac Implants. 1999;14:127-36. 